\title{
Análise de preferência declarada para estudar a utilidade da produção de oleaginosas
}

\author{
Francisco Roberto Farias Guimarães Júnior ${ }^{a *}$, Ana Augusta Ferreira de Freitas ${ }^{\mathrm{b}}$, \\ Luciana Gondim de Almeida Guimarãesc, Marcos André Mendes Primo ${ }^{d}$, \\ Márcio André Veras Machado ${ }^{\mathrm{e}}$ \\ a*rguimaraesjr@gmail.com, UFPB, Brasil \\ bfreitas8@terra.com.br, UECE, Brasil \\ clugondim@gmail.com, FMGR, Brasil \\ dmarcos.primo@ufpe.br, UFPE, Brasil \\ emavmachado@hotmail.com, UFPB, Brasil
}

\begin{abstract}
Resumo
0 objetivo deste trabalho foi elaborar um modelo de análise de preferência de escolha discreta e analisar a oferta de oleaginosas com base em dados de Preferência declarada, visto que a lei n. 11.097/2005 fixa a obrigatoriedade da introdução do biodiesel na matriz energética brasileira, o que garante a sua demanda. A metodologia foi do tipo descritiva e survey. 0 universo desta pesquisa foi composto por pequenos e médios produtores rurais, totalizando 114 observações válidas. 0 modelo desta pesquisa foi quantitativo, utilizando a análise de preferência declarada para analisar os dados. Dentre os principais resultados do estudo, verificou-se que os atributos que têm maior importância são: o retorno econômico e a existência de boa assistência técnica rural. A decisão dos produtores rurais não considerou a variável Teor de óleo importante. Uma das sugestões é aplicar o mesmo estudo para outras cidades e outros estados brasileiros.
\end{abstract}

Palavras-chave

Logística. Oleaginosas. Biodiesel. Preferência declarada.

\section{Introdução}

Estudos de previsão baseados tanto em métodos qualitativos (relacionados à experiência dos vendedores, por exemplo) quanto em métodos quantitativos, como os baseados em análises estatísticas e em séries temporais (CHEN; XIAO, 2009), têm sido realizados por vários pesquisadores. No entanto, os modelos tradicionais de previsão na óptica do produto focam a previsão de demanda. Isso se deve-se a que, com o mercado turbulento e em constante mudança, é necessário um ajuste em tempo real desta previsão para reduzir as incertezas e, consequentemente, a falta e/ou o excesso de produtos.

No caso do biodiesel, a incerteza de sua demanda praticamente não existe, pois a lei $n^{\circ} 11.097$ de 13 de janeiro de 2005, fixa, no caput do artigo $2^{\circ}$, a obrigatoriedade da introdução do biodiesel na matriz energética brasileira, sendo fixado em $5 \%$ o volume percentual mínimo obrigatório de adição dele ao óleo diesel comercializado ao consumidor final em qualquer parte do território nacional. 0 prazo para aplicação do disposto no caput desse artigo é de 8 anos (2013) após a publicação da lei, sendo de 3 anos (BRASIL, 2008) o período, após a publicação, para a adição de um percentual mínimo obrigatório intermediário de $2 \%$ de biodiesel, em volume, ao diesel comercializado.

Neste contexto, o governo brasileiro criou o Programa Nacional de Produção e Uso de Biodiesel (PNPB) através de decreto, em 23 de dezembro de 2003, instituindo a Comissão Executiva Interministerial, que é encarregada da implantação das ações direcionadas à produção e ao uso de óleo vegetal como fonte alternativa de energia (BRASIL, 2007). Equivalente ao Proálcool, o PNPB visa o desenvolvimento integrado e em rede das tecnologias de produção, industrialização e uso do biodiesel e de sua mistura com o diesel, a partir de óleos vegetais puros e residuais produzidos regionalmente. 
Observa-se, então, que essa lei impacta todas as empresas produtoras e distribuidoras de combustíveis que atuam no Brasil, como é o caso, por exemplo, da Petrobrás. Além disso, estimula o surgimento de novos players (indústrias, pequenas empresas e trabalhadores individuais) para atuarem nessa nova cadeia produtiva.

Assim, esta pesquisa parte do pressuposto de que os estudos de oferta de oleaginosas para produção de biodiesel são vitais, para se obter um equilíbrio para a sua demanda, que permita aumentar a escala de produção, executar um dimensionamento otimizado da infraestrutura logística e justificar a criação de novas fontes produtivas ou a ampliação das fontes existentes. Então, as informações resultantes deste estudo constituem uma das bases para a tomada de decisões quanto a investimentos na produção de oleaginosas, viabilizando estudos tecnológicos e programas de incentivo para a inclusão desse biocombustível na matriz energética brasileira e para o desenvolvimento do Brasil.

0 seguinte questionamento foi foco desta pesquisa: qual a importância e a utilidade dada pelos produtores às diferentes variáveis de decisão para cultivar oleaginosas para a produção de biodiesel?

Desta forma, o objetivo desta pesquisa é identificar a importância e a utilidade dada pelos produtores às diferentes variáveis de decisão condicionantes de investimentos na planta agrícola de oleaginosas para a produção de biodiesel, por meio de um modelo de escolha discreta com base em dados de preferência declarada (PD).

\section{0 processo de escolha dos indivíduos e o modelo de preferência declarada}

0 processo de escolha racional de indivíduos segue uma sequência caracterizada no fluxograma mostrado na Figura 1. Diante da necessidade de tomar uma decisão, um indivíduo coleta informações sobre as alternativas disponíveis e os valores dos atributos dessas alternativas. A seguir, compara as alternativas através de funções utilidades, forma sua preferência individual e avalia a alternativa escolhida (BEN-AKIVA; LERMAN, 1985; LOUVIERE; HENSHER; SWAIT, 2000).

Esse processo de escolha envolve, basicamente, as seguintes condições (LOUVIERE; HENSHER; SWAIT, 2000, p. 10):

- Dependem das características individuais e socioeconômicas do tomador de decisão (renda, idade, nível de escolaridade, etc.);

- As alternativas são constituídas por um conjunto de atributos que são avaliados pelo tomador de decisão no processo de escolha; e

- 0 indivíduo escolhe uma dentre as várias alternativas existentes com o objetivo de satisfazer ao máximo as suas necessidades.
0 item (c) está relacionado à Teoria microeconômica do consumidor, a qual se baseia na hipótese de que os indivíduos são racionais. Essa teoria supõe que existe uma medida dessa satisfação ou, pelo menos, uma escala ordinal de preferências na tomada de decisão. Dessa forma, o indivíduo procura se situar no ponto mais alto de sua escala de preferências. A medida de satisfação com relação a uma determinada alternativa é chamada de utilidade.

Estudos da Teoria da utilidade encontrados em Bentham (1871) afirmam que a motivação humana pode ser resumida em um único princípio: o desejo de maximizar a utilidade. Hoje,

[...] a moderna Teoria da utilidade, atribuída a John von Neumann e Oskar Morgenstern, cujos axiomas deram origem à Teoria da decisão, visa ser um retrato da atitude do indivíduo diante do risco [...] (ANTUNES; SANTOS, 2009, p. 286).

Como o processo de tomada de decisão dos produtores a respeito de investimentos na planta agrícola de oleaginosas é uma decisão empresarial, adotou-se, nesta pesquisa, o pressuposto de que esse processo de tomada de decisão é racional, o que suporta a utilização desse método de análise.

Segundo Lancaster (1966, apud BEN-AKIVA; LERMAN, 1985), a utilidade resultante de uma escolha individual pode ser definida em termos de atributos inerentes ao produto, ou seja, a função utilidade relaciona os valores dos atributos com a utilidade do produto de forma que essa utilidade seja uma função dos atributos do produto:

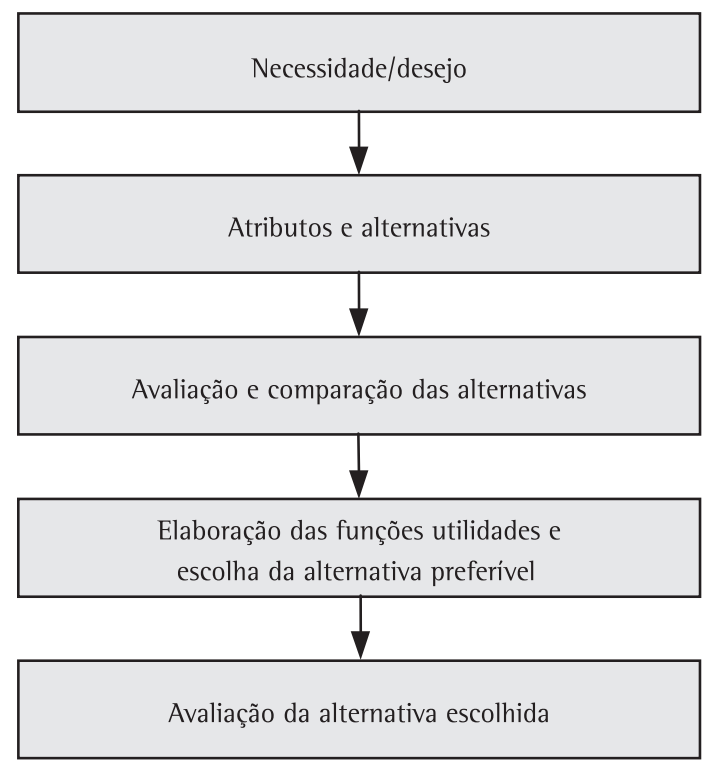

Figura 1. 0 processo de escolha dos consumidores. Fonte: Louviere, Hensher e Swait (2000, p. 8). 
$U=f\left(x_{1}, x_{2}, x_{3}, \ldots, x_{i}\right)$

onde:

- $U=$ Utilidade do produto $\mathrm{n}$;

- $x_{1}, x_{2}, x_{3}, \ldots, x_{i}=$ Atributos que caracterizam a utilidade do produto.

A partir dessa função, a qual associa atributos do produto à sua utilidade, é possível analisar o comportamento dos indivíduos diante de várias alternativas disponíveis. As informações adquiridas através de dados de PD e/ou de Preferência revelada (PR), podem ser utilizadas de várias formas: desde a estimativa da oferta e demanda de produtos existentes e de novos produtos, até a definição de políticas públicas (KROES; SHELDON, 1988; LOUVIERE; HENSHER; SWAIT, 2000).

Os métodos de PD mais conhecidos são: conjoint analysis (análise conjunta, normalmente, a mais utilizada), medida funcional, análise trade-off e método transfer-price. (HAIR JUNIOR et al., 2005). Nas técnicas de análise conjunta, o pesquisador elabora um conjunto de alternativas (hipotéticas ou não), as quais são representadas por um grupo de atributos com valores diferentes, de maneira que o efeito individual de cada atributo possa ser estimado. Isto é possível com a elaboração de um projeto experimental que assegure que as variações nos atributos em cada grupo sejam estatisticamente independentes umas das outras.

De acordo com Ortúzar e Willumsen (1994), um projeto experimental de PD do tipo análise conjunta deve ser feito em diferentes estágios, mostrados a seguir:

- Identificar os atributos-chave de cada alternativa e construir conjuntos de escolha. Todos os atributos essenciais devem estar presentes e as opções devem ser plausíveis e realistas;

- Projetar o experimento de maneira que as opções apresentadas aos respondentes facilitem expressar suas preferências. A forma de apresentação das alternativas deve ser fácil de entender e estar consoante com a experiência dos respondentes e suas restrições;

- Desenvolver uma estratégia de amostragem que assegure um bom e representativo conjunto de dados;

- Conduzir o levantamento de forma apropriada, incluindo supervisão e procedimentos de controle de qualidade; e

- Usar boas técnicas de estimação de modelos, sendo mais adequado e seguro, do ponto de vista de representação da realidade, a utilização conjunta de dados de PD e PR, o que aumenta a confiabilidade dos resultados (ARRUDA, 1995).

A análise conjunta é uma técnica que procura determinar a importância relativa que os entrevistados dão a atributos relevantes e a utilidade que eles associam aos níveis de atributos. A suposição é que qualquer conjunto de estímulos, como produtos, marcas ou lojas, seja avaliado como um pacote de atributos (PEDRET; SAGNIER; CAMP, 2000).

Em análise conjunta, os estímulos são combinações de níveis de atributos determinados pelo pesquisador. A análise conjunta procura estabelecer funções de valor parcial ou de utilidade parcial que descrevem o grau de utilidade que os consumidores associam aos níveis de cada atributo (HAIR JUNIOR et al., 2005).

Os dados de entrada em PD podem ser métricos ou não métricos (ORTÚZAR; WILLUMSEN, 1994). Para dados não métricos, os entrevistados devem formular avaliações por ordenação de postos (ranking). Na forma métrica, os entrevistados dão classificações em vez de ordenação por postos (rating). Nesse caso, os julgamentos se fazem de forma tipicamente independente.

Segundo Hensher (1994), existem três forma para o tomador de decisão refletir sobre suas preferências: ranking, rating e choice. Um experimento do tipo ranking parte do princípio de que o tomador de decisão tem capacidade de ordenar as alternativas de acordo com suas preferências. No experimento do tipo rating, o analista avalia as suas escolhas por meio de notas dadas às alternativas. Já no experimento do tipo choice, ou pairwise-choice, a escolha é feita por meio de uma comparação de duas alternativas. A vantagem do experimento do tipo choice está na simplicidade com a qual as alternativas são apresentadas aos respondentes, o que facilita o processo de escolha. Por esse motivo esta pesquisa utilizou o método choice.

A modelagem da estrutura da decisão pelo método choice é normalmente feita tendo por base o modelo logit (SENNA, 1994), haja vista que a variável dependente é não métrica e assume valores iguais a 1, quando a primeira opção é escolhida, e 0 no caso contrário. A forma funcional para o caso multinomial é:

$$
P_{i}=\frac{e^{U_{k}}}{\sum_{j=1}^{n} e^{U_{j}}}
$$

onde:

$P_{i}$ é a probabilidade da alternativa i ser escolhida e e é a base do logaritmo neperiano.

Admitindo-se que o termo aleatório da função utilidade seja regido por uma distribuição tipo Gumbel, chega-se ao modelo Logit multinomial que pode ser escrito como em Ben-Akiva e Lerman (1985):

$$
P_{n}(i)=\frac{e^{\beta_{k} x_{i n k}}}{\sum_{j \in C} e^{\beta_{k} x_{i n k}}}
$$


em que $P_{n}(i)$ é a probabilidade da alternativa i ser escolhida pelo indivíduo $\mathrm{n}$ dentro de um conjunto de possibilidades $C, \beta_{k}$ são os parâmetros a serem estimados do modelo e $X_{\text {jnk }}$ são atributos que caracterizam a função utilidade.

Uma propriedade-chave dos modelos de escolha estocásticos, que é o caso do logit multinomial, é o princípio da independência entre as alternativas irrelevantes. Na prática, em uma amostra de 100 observações em que 80 escolhem a alternativa A e 20 escolhem a alternativa B, espera-se a mesma desigualdade de escolha (isto é, $80 / 20=4$ ), se os mesmos escolhessem entre as alternativa A, B e C, ou seja, 60, 15 e 25 , respectivamente $(60 / 15=4)$ (FREITAS, 1995).

Modelos de escolha discreta são modelos probabilísticos desagregados que têm como forma usual o modelo Logit. Esse tipo de análise envolve cálculos complexos e requer softwares específicos como o ALOGIT 4.1, desenvolvido pelo Hague Consulting Group (2004) e utilizado neste trabalho e Como já mencionado antes, utilizou-se o software ALOGIT 4.1 do Hague Consulting Group para o cálculo dos parâmetros da Equação 5.

\section{Metodologia da pesquisa}

Quanto aos procedimentos, trata-se de uma pesquisa do tipo bibliográfica e de survey. Quanto aos objetivos, a metodologia foi do tipo descritiva, pois o trabalho foi desenvolvido no sentido de descrever a importância e a utilidade dada pelos produtores às diferentes variáveis de decisão condicionantes de investimentos no cultivo de culturas oleaginosas (CERVO; BERVIAN, 1983).

No que se refere à abordagem do problema, esta pesquisa foi quantitativa. A técnica utilizada na elaboração do modelo foi a análise de preferência declarada, que é uma técnica que investiga as preferências dos entrevistados e baseia-se em intenções. 0 suporte teórico para a utilização dessa técnica é o fato de o processo de tomada de decisão dos produtores rurais ser uma decisão empresarial, o que permite adotar o pressuposto de que esse processo de tomada de decisão é racional.

Para identificar os principais atributos analisados por meio da técnica de PD, utilizaram-se os resultados encontrados por Guimarães (2008). Em seu trabalho, que teve como objetivo

[...] identificar quais as principais variáveis e o seu grau de importância para a tomada de decisão sobre investimentos em plantas agrícolas para o cultivo de oleaginosas no âmbito da cadeia produtiva do biodiesel [...] (GUIMARÃES, 2008, p. 24)

foi aplicada uma análise fatorial (HAIR JUNIOR et al., 2005) para calcular o grau de importância individual de cada variável e agrupá-las por meio de suas comunalidades, dando origem a novos constructos.

Analisando, na Tabela 1, os resultados de Guimarães (2008), verifica-se que 15 das 21 primeiras variáveis de decisão são relacionadas com recursos financeiros, apoio recebido na forma de assistência técnica e teor de óleo extraído da oleaginosa.

Dessa forma, elaborou-se o questionário deste trabalho levando-se em consideração que nele deveriam constar perfis com os atributos (variáveis) apresentados na Tabela 2: oleaginosa, retorno econômico, existência de boa assistência técnica rural e bom teor de óleo. 0 atributo "oleaginosa", que não se encontra na Tabela 1, foi considerado nesse questionário porque em algumas situações o agricultor escolhe o que plantar com base apenas no tipo de cultura, desconsiderando outros aspectos (MENDES, 2005; PERES; BELTRÃO, 2006).

Uma função utilidade foi gerada, contendo as variáveis: oleaginosas; retorno econômico; existência de boa assistência técnica; e teor de óleo, a qual está apresentada na Equação 4. Como oleaginosas é uma variável nominal ela precisou ser codificada como variável dummy. Pelo fato dessa variável apresentar três níveis (mamona, girassol e amendoim), foi codificada com duas dummies, que nesse caso são $x_{1}$ e $x_{2}$, onde 10 significa mamona, 01 , amendoim e 00 , girassol. Já a variável existência de boa assistência técnica foi codificada com uma dummy por apresentar dois niveis (CARMO; DIAZ, 2000, p. 91). Vale ressaltar que se mudarmos a codificação das variáveis, os resultados não modificam. Como as variáveis retorno econômico e teor de óleo são métricas, dispensa a codificação em dummies.

$$
\begin{aligned}
& U(x)=\beta_{1} \times O L\left(x_{1}\right)+\beta_{2} \times O L\left(x_{2}\right)+ \\
& \beta_{3} \times R E+\beta_{4} \times A T+\beta_{5} \times T O
\end{aligned}
$$

\section{sendo:}

- $\mathrm{U}(\mathrm{x})=$ Função utilidade para analisar a preferência dos agricultores;

- $\mathrm{OL}\left(\mathrm{x}_{\mathrm{i}}\right)=$ Valor do nível do atributo oleaginosa;

- $\mathrm{RE}=$ Valor do nível do atributo retorno econômico; AT $=$ Valor do nível do atributo existência de boa assistência técnica;

- $\mathrm{TO}=$ Valor do nível do atributo teor de óleo;

- $\beta_{\mathrm{j}}=$ Coeficientes que serão estimados para se encontrar os valores das utilidades.

Para a definição dos níveis de cada atributo, utilizaram-se os seguintes critérios:

- Para o atributo oleaginosa, discutiu-se na reunião do pré-teste (explicada com mais detalhes no item 3.1) quais eram as culturas com maior aceitação por parte dos produtores rurais no estado do Ceará;

- Com relação à existência de boa assistência técnica, as respostas só podiam ser sim ou não; e 
Tabela 1. Grau de importância das variáveis de decisão.

\begin{tabular}{|c|c|}
\hline Variáveis de decisão & Grau de importância \\
\hline Benefícios aos cooperados & 0,742 \\
\hline Recursos imobilizados e em caixa & 0,687 \\
\hline Nível de apoio dos cooperados à administração da cooperativa & 0,641 \\
\hline Adimplência de produtores e cooperativas no BB e no BNB & 0,627 \\
\hline Existência de assessoria de nível superior & 0,553 \\
\hline Parcerias com Centec & 0,456 \\
\hline Disponibilidade de crédito rural & 0,404 \\
\hline Assistência da Embrapa & 0,398 \\
\hline Qualidade da infraestrutura de energia & 0,330 \\
\hline Crédito de carbono & 0,296 \\
\hline Existência de fórum de assentados & 0,276 \\
\hline Cumprimento das orientações técnicas & 0,251 \\
\hline Infraestrutura de armazenamento registrado na Conab & 0,243 \\
\hline Área plantada nos assentamentos & 0,234 \\
\hline Existência de agências do BNB e do BB & 0,220 \\
\hline Assistência do lncra & 0,220 \\
\hline Distribuição de sementes pelo governo & 0,219 \\
\hline Número de assentamentos & 0,216 \\
\hline Canal efetivo de comunicação entre agricultores familiares e técnicos da Ater & 0,204 \\
\hline Nivel de organização dos produtores familiares em associações e/ou cooperativas & 0,179 \\
\hline Teor de óleo extraído & 0,174 \\
\hline Proximidade e uso de açudes públicos & 0,165 \\
\hline Mão de obra qualificada & 0,144 \\
\hline Município zoneado pela Embrapa & 0,143 \\
\hline Período necessário para início de colheita & 0,139 \\
\hline Qualidade da infraestrutura de instalações & 0,138 \\
\hline Possibilidade de retorno ao cultivo de oleaginosas no curto-médio prazo & 0,129 \\
\hline Número de famílias assentadas & 0,125 \\
\hline Interesse da comunidade & 0,108 \\
\hline Avaliação dos serviços prestados à cooperativa pelos agricultores & 0,103 \\
\hline Estado de conservação de rodovias e ferrovias & 0,103 \\
\hline Infraestrutura pública (hospital, escola) & 0,098 \\
\hline Proximidade das fontes de matéria-prima (sementes) & 0,097 \\
\hline Proximidade do Centec & 0,074 \\
\hline Existência de escritórios de Ater pública/privada aptos à estruturação de propostas de financiamento & 0,065 \\
\hline Área mínima necessária para plantio & 0,042 \\
\hline Proximidade de rodovias e ferrovias (estação) & 0,041 \\
\hline Venda garantida & 0,033 \\
\hline Distância em relação às usinas de esmagamento & 0,022 \\
\hline Capacidade efetiva de armazenagem & 0,004 \\
\hline Tradição em cultivo de mamona e de outras oleaginosas & 0,001 \\
\hline Município zoneado & $-0,007$ \\
\hline Incentivo fiscal & $-0,022$ \\
\hline Ambientalmente correto & $-0,022$ \\
\hline Qualidade da infraestrutura de acesso às usinas (esmagamento/beneficiamento) & $-0,028$ \\
\hline Produtividade de óleo & $-0,029$ \\
\hline Resistência a pragas & $-0,046$ \\
\hline
\end{tabular}

Fonte: Guimarães (2008).

- Para os atributos retorno econômico e teor de óleo, utilizaram-se informações constantes nos relatórios semanais disponiveis no site da Companhia Nacional de Abastecimento (Conab), e na home page da Empresa Brasileira de Pesquisa Agropecuária (Embrapa).
Quando o experimento envolve o estudo dos efeitos de dois ou mais fatores (atributos), a estratégia mais eficiente é o planejamento fatorial. Nessa estratégia, os fatores são variados juntos e todas as possíveis combinações dos níveis dos fatores são investigadas (MONTGOMERY, 2001). 
Tabela 2. Atributos e níveis de atributos.

\begin{tabular}{ll}
\hline \multicolumn{1}{c}{ Atributo } & \multicolumn{1}{c}{ Nivel } \\
\hline \multirow{3}{*}{ Oleaginosa } & Mamona \\
& Girassol \\
& Amendoim \\
& $\mathrm{R} \$ 48,00$ por $60 \mathrm{~kg}$ de baga \\
& $\mathrm{R} \$ 40,00$ por $60 \mathrm{~kg}$ de baga \\
Retorno econômico & $\mathrm{R} \$ 25,00$ por $60 \mathrm{~kg}$ de baga \\
& $\mathrm{R} \$ 20,00$ por $60 \mathrm{~kg}$ de baga \\
Existência de boa assistência técnica & $\mathrm{Sim}$ \\
& $\mathrm{Não}$ \\
Teor de óleo & 0,40 \\
& 0,45 \\
& 0,50 \\
\hline
\end{tabular}

Fonte: Elaborada pelos autores.

Dessa forma, pode-se estimar os efeitos de um fator nos diversos níveis dos outros fatores, conduzindo a conclusões que são válidas dentro da abrangência das condições experimentais, essa a única forma de descobrir interações entre os fatores, evitando conclusões incorretas quando houver interações entre fatores (MONTGOMERY, 2001). 0 problema central num planejamento fatorial é o crescimento exponencial das combinações de níveis de atributos com o aumento do número de fatores (atributos).

0 planejamento fatorial fracionário fornece um meio de obter boas estimativas dos efeitos principais e das interações de segunda ordem com uma fração do esforço requerido por um planejamento fatorial completo. Um planejamento fatorial fracionado é construído selecionando um subconjunto das combinações de um planejamento fatorial completo e executando a análise neste subconjunto escolhido (MONTGOMERY, 2001).

Logo, com os quatro atributos desta pesquisa definidos em vários níveis, pôde-se construir um total de 72 perfis $(3 \times 4 \times 2 \times 3)$. Para facilitar a avaliação por parte do entrevistado, empregou-se um planejamento fatorial fracionado, construindo-se um subconjunto de 10 perfis para os estímulos de estimação, onde os entrevistados escolheram a preferência para o plantio. A escolha desses perfis foi aleatória, tendo como único critério a existência de todos os níveis de todos os atributos nos 10 perfis escolhidos, o que possibilitou compará-los entre si.

Ressalta-se a possibilidade de não ser possível avaliar todos os efeitos conjuntos de todos os fatores dado que só estão presentes na amostra nalgumas combinações. Mas, pelo princípio da independência entre as alternativas irrelevantes espera-se que a razão entre as escolhas permaneça constante, o que torna mínima as perdas provocadas pelo planejamento fatorial fracionado na avaliação de todos os efeitos conjuntos de todos os fatores.

\subsection{Universo e amostra}

0 universo desta pesquisa foi composto pelos pequenos e médios produtores rurais do estado do Ceará com experiência no setor de produção de oleaginosas e que produziam na região do semiárido. A amostra foi por acessibilidade e totalizou 114 observações válidas (questionários), adequando-se ao proposto por Hair Junior et al. (2005, p. 98), que afirma que para pesquisas quantitativas com análises multivariadas o tamanho da amostra deve ter pelo menos cinco vezes mais observações que o número de variáveis a serem analisadas. Alguns pesquisadores chegam a propor um mínimo de vinte casos para cada variável.

A área estudada foi constituída pelos seguintes municípios que fazem parte da região do semiárido cearense: Canindé, Itapipoca, Quixadá, Quixeramobim e Sobral. Esses municípios tiveram como critério de escolha a amostra por julgamento (intencional). De acordo com Malhotra (2006, p. 327), “[ ...] os elementos da população são selecionados deliberadamente com base no julgamento do pesquisador [...]".

Os pesquisadores escolheram as localidades em conjunto com o grupo de trabalho Biodiesel e inclusão social, coordenado pelo DNOCS, o qual as considerou cidades-chave na extração de óleo no Ceará, pois expressam o potencial produtivo e de esmagamento (extração de óleo) de oleaginosas no estado.

\subsection{Planejamento da pesquisa de campo}

0 instrumento de coleta de dados utilizado foi um questionário estruturado, contendo dez perfis de resultado produtivo da plantação de cada cultura. Esses perfis foram combinados em seis cenários de escolhas com dois perfis em cada, pois o método escolhido para análise foi o choice. A escolha das combinações dos perfis para criação do cenário foi aleatória. Nota-se que dois perfis são repetidos em cenários distintos ( 6 cenários $\times 2$ perfis $=12$ perfis).

Foi realizado um pré-teste em um grupo de 15 respondentes especialistas na área, no dia $11 \mathrm{de}$ junho de 2009, na sede do Departamento Nacional de Obras Contra a Seca (DNOCS), onde se verificou dificuldade de compreensão e análise dos perfis (alternativas). Os 15 especialistas eram dois do DNOCS, dois da Universidade Federal do Ceará, um da Fundação Núcleo de Tecnologia Industrial do Ceará (Nutec), um do Banco do Nordeste, um do Banco do Brasil, dois da Petrobrás, um da Federação dos Trabalhadores e Trabalhadoras na Agricultura do Estado do Ceará (Fetraece), um da Federação dos Trabalhadores na Agricultura Familiar (Fetraf), um da Empresa de Assistência Técnica e Extensão Rural do Ceará (Ematerce), um do Instituto Centro de Ensino Tecnológico (Centec), um do Movimento 
dos Trabalhadores Sem Terra (MST) e um do Instituto Nacional de Colonização e Reforma Agrária (Incra). A seguir, fez-se uma pesquisa piloto, com o objetivo de eliminar as falhas do questionário e ajustar a pesquisa ao vocabulário e à realidade dos produtores rurais.

A pesquisa piloto foi realizada na cidade de Canindé, CE, em 20 de junho de 2009, com os pequenos e médios produtores rurais. Nela foram aplicados 34 questionários, obtendo-se 28 válidos. 0 resultado da pesquisa piloto apontou que os produtores compreenderam o objetivo da pesquisa e responderam o questionário com facilidade. Escolheu-se Canindé por ser, dentre as cinco cidades escolhidas, a mais próxima de Fortaleza.

\subsection{Tratamento dos dados}

Primeiramente, foi feita uma análise descritiva, a fim de verificar sexo e município dos respondentes. 0 segundo passo foi a aplicação do método de análise de dados de PD para identificar a utilidade de cada variável e a importância dos níveis de cada atributo na percepção dos agricultores quanto ao investimento no cultivo de oleaginosas para a cadeia do biodiesel. Com relação ao sexo, o masculino representou 73\%, enquanto o feminino, 27\%. No que diz respeito ao município, em Canindé foram 32 questionários, em Itapipoca, 19, em Quixadá, 25, em Quixeramobim, 23 e, em Sobral, 15 questionários.

A aplicação do modelo multinominal Logit para a análise de dados de PD foi feita por meio da equação verossimilhança apresentada na Equação 5, pois as variáveis são idênticas e independentemente distribuídas (BEN-AKIVA; LERMAN, 1985, p. 20).

$$
(\theta)=f(x ; \theta)=\prod_{i=1}^{n} f\left(x_{i} ; \theta_{i}\right)
$$

onde:

- $x_{i}=$ São as variáveis utilizadas no modelo;

- $\theta_{i}=\left(\mu, \sigma^{2}\right), i=1, \ldots$, n é o vetor de parâmetros da distribuição $f(x)$ com média $\mu$ e variância $\sigma^{2}$.

Como já mencionado antes, utilizou-se o software ALOGIT 4.1 do Hague Consulting Group (2004) para o cálculo dos parâmetros da Equação 5 .

Tabela. 3. Matriz de correlação de Pearson das estimativas dos parâmetros do modelo.

\begin{tabular}{lcccc} 
& $\hat{\beta}_{1}$ & $\hat{\beta}_{2}$ & $\hat{\beta}_{3}$ & $\hat{\beta}_{4}$ \\
\hline$\hat{\beta}_{2}$ & 0,304 & & & \\
$\hat{\beta}_{3}$ & $-0,730$ & $-0,024$ & & \\
$\hat{\beta}_{4}$ & $-0,347$ & $-0,189$ & 0,347 & \\
$\hat{\beta}_{5}$ & $-0,103$ & $-0,268$ & 0,220 & $-0,100$ \\
\hline
\end{tabular}

Fonte: Cálculo dos autores.

\section{Análise dos dados e interpretação dos resultados}

A primeira informação que deve ser analisada no arquivo de saída do software é a matriz de correlação de Pearson das estimativas dos parâmetros do modelo da Equação 4, para verificar se há consistência nos resultados. Essa matriz está apresentada na Tabela 3 e, para sua análise, adotaram-se os mesmos critérios utilizados por Brandão Filho (2005):

- Correlação forte: $0,70<|\rho| \leq 1$;

- Correlação moderada: 0,50< $|\rho| \leq 0,70$;

- Correlação fraca: $0<|\rho| \leq 0,50$; e

- Ausência de correlação: $\rho=0$.

Dessa forma, conforme Tabela 3, tem-se que existe apenas uma correlação forte entre $\hat{\beta}_{1}$ e $\hat{\beta}_{3}(-0,730)$ entre as estimativas dos parâmetros do modelo, o que não invalida a pesquisa e dá consistência ao modelo, visto que todas as outras correlações apresentaram valores entre $-0,35$ e 0,35 e a correlação forte entre $\hat{\beta}_{1}$ e $\hat{\beta}_{3}$ pôde ser explicada.

Esta correlação forte entre $\hat{\beta}_{1}$ e $\hat{\beta}_{3}$ pode ser um reflexo da política de incentivo ao plantio de mamona realizada pelo governo do estado em parceria com o governo federal, haja vista que $\beta$ é o coeficiente da dummy que representa mamona e $\beta_{3}$ é o coeficiente de retorno econômico. Essa política garante a compra da tonelada de mamona por um preço mínimo de $\mathrm{R} \$ 0,70$, complementado com o pagamento de um auxílio ao plantio de $\mathrm{R} \$ 150,00$ por hectare no ato da plantação, limitado a três hectares ( $R \$ 450,00)$, se o agricultor implantar essa cultura. Segundo Morikawa (1989), são comuns algumas correlações moderadas e fortes em experimentos de PD, haja vista a dificuldade de se planejar um experimento.

0 segundo parâmetro analisado foi o pseudocoeficiente de determinação ( $\left.\rho^{2}\right)$, o qual mede o grau de ajustamento da equação encontrada aos dados originais e tem um valor teórico limitado de 0 a 1 . Valores acima de 0,2 e próximo a 0,4 indicam um ajuste considerado apropriado para o modelo Logit multinomial, segundo Ortúzar e Willumsen (1994).

Nesse caso é observado na Tabela 4 um valor de $\rho^{2}$ igual a 0,2059 para o modelo w.r.t. Zero e $\rho^{2}$ igual a 0,2040 para o modelo w.r.t. Constants, indicando que as variáveis independentes se ajustam bem ao modelo.

Observam-se nessa Tabela 4 dois modelos: w.r.t. Zero e w.r.t. Constants. A sigla w.r.t. significa with

Tabela 4. Pseudocoeficientes de determinação do modelo.

\begin{tabular}{lcc}
\multicolumn{1}{c}{ Modelo } & $\rho$ & $\rho^{2}$ \\
\hline w.r.t. Zero & 0,453762 & 0,2059 \\
w.r.t. Constants & 0,451664 & 0,2040 \\
\hline Fonte: Cálculo dos autores. & &
\end{tabular}


Tabela 5. Valores dos $\rho^{2}$ de pesquisas que utilizaram a metodologia de preferência declarada.

\begin{tabular}{lll}
\hline \multicolumn{1}{c}{ Autores } & Local da publicação & Valor do $\rho^{2}$ \\
\hline Haaijer, Kamakura e Wede (2000) & Journal of Marketing Research & Modelo $1=0,1970$ \\
& & Modelo $2=0,2900$ \\
Brito (2007) & Modelo $3=0,2980$ \\
& POLI/USP (Mestrado em Engenharia de Transportes) & Modelo $1=0,4313$ \\
Alves (2005) & Modelo $2=0,4383$ \\
& POLI/USP (Mestrado em Engenharia de Transportes) & Modelo $1=0,0276$ \\
Brandão Filho (2005) & Modelo $2=0,0331$ \\
& Petran/UFC (Mestrado em Engenharia de Transportes) & Modelo $3=0,0327$ \\
\end{tabular}

Fonte: Elaborada pelos autores.

ratio test, a qual denota que o $\rho^{2}$ foi calculado com base no teste da razão de erossimilhavnça (Likelihood Ratio Test). Os termos Zero e Constants são utilizados para indicar, respectivamente, o $\rho^{2}$ e o $\rho^{2}$ ajustado que também pode ser expresso como $\bar{\rho}^{2}$ e é utilizado para verificar se a adição de mais variáveis ao modelo é realmente necessária, apesar do aumento no valor de $\rho^{2}$ e para comparar modelos com diferentes números de variáveis (BEN-AKIVA; LERMAN, 1985, p. 167). As equações para o cálculo de $\rho^{2}$ e $\rho^{2}$ ajustado são:

$\rho^{2}=1-\frac{\mathscr{L}(\hat{\beta})}{\mathscr{L}(0)}$

$\rho_{\text {ajustado }}^{2}=1-\frac{\mathscr{L}(\hat{\beta})-K}{\mathscr{L}(0)}$

onde:

- $\mathscr{L}(\hat{\beta})=\log$ verossimilhança do modelo considerando os parâmetros estimados;

- $\mathscr{L}(0)=\log$ verossimilhança do modelo considerando os parâmetros nulos;

- $\mathscr{L}(\hat{\beta})-K=$ é o critério de informação de Akaike. É necessário subtrair $\mathrm{K}$ para remover o efeito de avaliação de $\mathscr{L}(\hat{\beta})$ nos valores anteriormente estimados para os verdadeiros parâmetros.

A Tabela 5 apresenta algumas pesquisas que utilizaram a metodologia de preferência declarada e os valores dos $\rho^{2}$. 0 propósito desta tabela é comparar o resultado do $\rho^{2}$ encontrado no modelo proposto por esta pesquisa com os resultados dos $\rho^{2}$ de outras pesquisas.

A próxima estatística analisada foi o teste da razão de verossimilhança (Likelihood Ratio Test), cuja função é: $L R=-2\{\mathscr{L}(0)-\mathscr{L}(\hat{\beta})\}$ e tem distribuição de $\chi^{2}$ com $r$ graus de liberdade, onde $r$ é o número de restrições lineares (parâmetros $\beta$ ). Essa estatística testa a hipótese de nulidade de todos
Tabela 6. Valor do teste LR e o score do $\chi_{(0,999 ; 9) \text {. }}^{2}$

\begin{tabular}{ccc}
\hline LR & $\chi^{2}$ & \multicolumn{1}{c}{ Resultado do teste de hipótese } \\
\hline 195,275 & 20,516 & $\begin{array}{l}\text { Rejeita-se a hipótese de nulidade de todos } \\
\text { os parâmetros simultaneamente }\end{array}$ \\
\hline
\end{tabular}

Fonte: Cálculo dos autores.

os parâmetros simultaneamente. Se o valor LR for maior que o valor $\chi_{(\alpha ;) \text {, }}^{2}$ então se rejeita a hipótese de nulidade de todos os parâmetros simultaneamente (BEN-AKIVA; LERMAN, 1985, p. 165). A Tabela 6 evidencia o valor do teste e o score do $\chi_{(0,999 ; 5)}^{2}$, ou seja, o valor do $\chi^{2}$ com 99,9\% de confiança e 5 graus de liberdade.

Com base nesses valores da Tabela 6 , rejeita-se a hipótese de nulidade de todos os parâmetros simultaneamente. Dessa forma, como os parâmetros $\beta$ estimados no modelo não são nulos, utilizam-se esses parâmetros, os quais estão apresentados na Tabela 7, para calcular as utilidades de cada alternativa de escolha, por meio das funções utilidades de cada alternativa, como apresentado por Louviere, Hensher e Swait (2000).

A função utilidade, que foi apresentada anteriormente na Equação 4, está descrita a seguir, na Equação 8, com os parâmetros estimados.

$$
\begin{aligned}
& U(x)=0,3182 \times O L\left(x_{1}\right)-0,3024 \times O L\left(x_{2}\right)+ \\
& 0,04211 \times R E+0,8845 \times A T+3,276 \times T O
\end{aligned}
$$

De acordo com os resultados da Tabela 8, considerando-se um nível de significância de 0,075 , todos os parâmetros estimados pelo modelo de PD foram significativos. Como as correlações foram baixas (todas as correlações foram entre $-0,35$ e 0,35 , exceto entre $\beta_{1}$ e $\beta_{3}$, cujo valor foi $-0,730$ ), o modelo é aceitável. Diante disso todos os coeficientes calculados no modelo são válidos.

A Tabela 8 apresenta os resultados das utilidades parciais e da importância, calculados através do modelo Logit multinomial. Esses resultados indicam a utilidade de cada nível dos atributos dos modelos e 
Tabela 7. Estimativas dos parâmetros do modelo multinomial Logit.

\begin{tabular}{lcccc}
\hline \multicolumn{1}{c}{ Variáveis } & $\hat{\beta}$ & $\begin{array}{c}\text { Erro } \\
\text { padrão }\end{array}$ & $\begin{array}{c}\text { Estatística } \\
\boldsymbol{t}\end{array}$ & $\boldsymbol{p}$-valor \\
\hline Oleaginosas $\left(\mathrm{X}_{1}\right)$ & 0,31820 & 0,1840 & 1,7 & 0,074938 \\
Oleaginosas $\left(\mathrm{X}_{2}\right)$ & $-0,30240$ & 0,1330 & $-2,3$ & 0,034886 \\
Retorno econômico & 0,04211 & 0,0117 & 3,6 & 0,007772 \\
Existência de boa & 0,88450 & 0,1130 & 7,8 & 0,000277 \\
assistência técnica & & & & \\
Teor de óleo & 3,27600 & 1,4600 & 2,2 & 0,039547 \\
\hline
\end{tabular}

Fonte: Cálculo dos autores.

Tabela 8. Resultados.

\begin{tabular}{lccc}
\hline \multicolumn{1}{c}{ Atributo } & Níveis & Utilidade parcial & Importância \\
\hline \multirow{3}{*}{ Oleaginosas } & Mamona & 0,318 & \\
& Girassol & 0,000 & 0,206 \\
& Amendoim & $-0,302$ & \\
& $\mathrm{R} \$ 48,00$ & 2,020 & \\
Retorno & $\mathrm{R} \$ 40,00$ & 1,680 & \multirow{2}{*}{0,3913} \\
econômico & $\mathrm{R} \$ 25,00$ & 1,050 & \\
& $\mathrm{R} \$ 20,00$ & 0,840 & \\
Existência de boa & Sim & 0,885 & 0,2935 \\
assistência técnica & $\mathrm{Não}$ & 0,000 & \\
& $50 \%$ & 1,640 & \multirow{2}{*}{0,1088} \\
Teor de óleo & $45 \%$ & 1,470 & \\
& $40 \%$ & 1,310 & \\
\hline
\end{tabular}

Fonte: Cálculo dos autores.

a importância relativa de cada atributo. Vale ressaltar que esses dois itens devem ser analisados em conjunto.

Através dos resultados obtidos, percebe-se quais os atributos mais importantes para os produtores e os níveis desses atributos que têm mais aceitação. Pode-se destacar a variável retorno econômico como o atributo que mais influencia no processo de escolha dos produtores quanto ao plantio de oleaginosas, visto que a sua importância foi superior à importância dos outros atributos.

Analisando-se as utilidades parciais desse atributo, verifica-se que os produtores rurais têm preferência por um retorno financeiro maior, indo ao encontro do exposto por Brigham e Ehrhardt (2002), os quais afirmam que em um investimento qualquer um dos objetivos do investidor é maximizar a taxa interna de retorno, que é a taxa de desconto que iguala o valor presente líquido a zero. $0 u$ seja, o investimento é pago em um menor tempo, pois se a taxa interna de retorno é aumentada, o payback, que é o prazo que o investimento retorna ao investidor, diminui.

A segunda variável mais importante no processo de escolha é a existência de boa assistência técnica. Percebe-se, então, que os produtores têm preferência por investimentos que sejam assistidos por profissionais capazes de lhes oferecer conhecimento técnico, o que
Tabela 9. Cálculo das utilidades parciais de cada nível dos atributos do modelo.

\begin{tabular}{|c|c|c|c|c|}
\hline Atributo & $\begin{array}{c}\beta \\
\text { calculado }\end{array}$ & $\begin{array}{c}\text { Operador } \\
\text { matemático }\end{array}$ & Niveis & $\begin{array}{c}\text { Utilidade } \\
\text { parcial }\end{array}$ \\
\hline \multirow{6}{*}{ Oleaginosas } & 0,31820 & \multirow{2}{*}{$x$} & 1 & \multirow{2}{*}{0,3182} \\
\hline & $-0,30240$ & & 0 & \\
\hline & 0,31820 & \multirow{2}{*}{$x$} & 0 & \multirow{2}{*}{0,0000} \\
\hline & $-0,30240$ & & 0 & \\
\hline & 0,31820 & \multirow{2}{*}{$x$} & 0 & \multirow{2}{*}{$-0,3024$} \\
\hline & $-0,30240$ & & 1 & \\
\hline \multirow{4}{*}{$\begin{array}{l}\text { Retorno } \\
\text { econômico }\end{array}$} & \multirow{4}{*}{0,04211} & \multirow{4}{*}{$x$} & $\mathrm{R} \$ 48,00$ & 2,020 \\
\hline & & & $\mathrm{R} \$ 40,00$ & 1,680 \\
\hline & & & $\mathrm{R} \$ 25,00$ & 1,050 \\
\hline & & & $\mathrm{R} \$ 20,00$ & 0,840 \\
\hline \multirow{2}{*}{$\begin{array}{l}\text { Existência de boa } \\
\text { assistência técnica }\end{array}$} & \multirow{2}{*}{0,88450} & \multirow{2}{*}{$x$} & $\operatorname{Sim}(1)$ & 0,885 \\
\hline & & & Não (0) & 0,000 \\
\hline \multirow{3}{*}{ Teor de Óleo } & \multirow{3}{*}{3,27600} & \multirow{3}{*}{$x$} & $50 \%$ & 1,640 \\
\hline & & & $45 \%$ & 1,470 \\
\hline & & & $40 \%$ & 1,310 \\
\hline
\end{tabular}

Fonte: Elaborada pelos autores.

demonstra o anseio que eles têm de trabalhar da forma correta, seguindo as orientações dos especialistas.

Na sequência da interpretação dos resultados, verifica-se que a terceira variável em nível de importância para a tomada de decisão dos produtores rurais é o tipo de oleaginosa. Existe uma maior preferência por plantar mamona, tendo o girassol como segunda opção e o amendoim como terceira. Esse resultado pode ser um reflexo da política de incentivo ao plantio de mamona feita pelo governo do estado em parceria com o governo federal, a qual tem como característica a garantia de compra por um preço mínimo de $\mathrm{R} \$ 0,70$ por tonelada. Além disso, esse programa é complementado com o pagamento de um auxílio ao plantio de $\mathrm{R} \$ 150,00$ por hectare no ato da plantação, limitado em até 3 hectares ( $R \$ 450,00)$, se o agricultor implantar esta cultura.

A variável teor de óleo apresentou valores baixos de grau de importância. lsso pode indicar que o teor de óleo não é uma variável-chave na tomada de decisão de investimentos em oleaginosas ou pode ter havido um problema no delineamento do experimento, pois os níveis desse atributo são muito próximos $(0,50 ; 0,45 ; 0,40)$. É importante destacar que esses percentuais de teor de óleo são informações técnicas e foram extraídas do site da Embrapa.

Uma conclusão é que se houver políticas de incentivo ao cultivo de oleaginosas com existência de boa assistência técnica rural (Ater), a produção de matéria-prima para as usinas extratoras aumentará consideravelmente. Quando de programas de incentivo, o tipo de oleaginosa que se deve focar deve ser a mais adaptada à região onde será plantada.

Os resultados evidenciados na Tabela 8 foram calculados de acordo com o procedimento descrito por Hair Junior et al. (2005, p. 324) e Malhotra (2006, 
Tabela 10. Cálculo da importância relativa de cada atributo do modelo.

\begin{tabular}{|c|c|c|c|c|c|c|}
\hline Atributo & Niveis & $\begin{array}{l}\text { Utilidades aarciais } \\
\text { máximas e mínimas }\end{array}$ & Ref. & Importância absoluta & Ref. & lmportância relativa \\
\hline \multirow{2}{*}{ Oleaginosas } & Mamona & 0,3182 & A & \multirow{2}{*}{$A-B=0,62$} & \multirow{2}{*}{1} & \multirow{2}{*}{$1 \div(1+J+K+L)=0,206$} \\
\hline & Amendoim & $-0,3024$ & B & & & \\
\hline \multirow{2}{*}{ Retorno econômico } & $\mathrm{R} \$ 48,00$ & 2,020 & C & \multirow{2}{*}{$C-D=1,18$} & \multirow{2}{*}{$\mathrm{J}$} & \multirow{2}{*}{$\mathrm{J} \div(1+\mathrm{J}+\mathrm{K}+\mathrm{L})=0,3913$} \\
\hline & $\mathrm{R} \$ 20,00$ & 0,840 & $\mathrm{D}$ & & & \\
\hline \multirow{2}{*}{$\begin{array}{l}\text { Existência de boa } \\
\text { assistência técnica }\end{array}$} & Sim & 0,885 & $\mathrm{E}$ & \multirow{2}{*}{$F-E=0,88$} & \multirow{2}{*}{ K } & \multirow{2}{*}{$K \div(1+J+K+L)=0,2935$} \\
\hline & Não & 0,000 & $\mathrm{~F}$ & & & \\
\hline \multirow{2}{*}{ Teor de óleo } & $50 \%$ & 1,640 & $\mathrm{G}$ & \multirow{2}{*}{$G-H=0,33$} & \multirow{2}{*}{$\mathrm{L}$} & \multirow{2}{*}{$\mathrm{L} \div(1+\mathrm{J}+\mathrm{K}+\mathrm{L})=0,1094$} \\
\hline & $40 \%$ & 1,310 & $\mathrm{H}$ & & & \\
\hline
\end{tabular}

Fonte: Elaborada pelos autores.

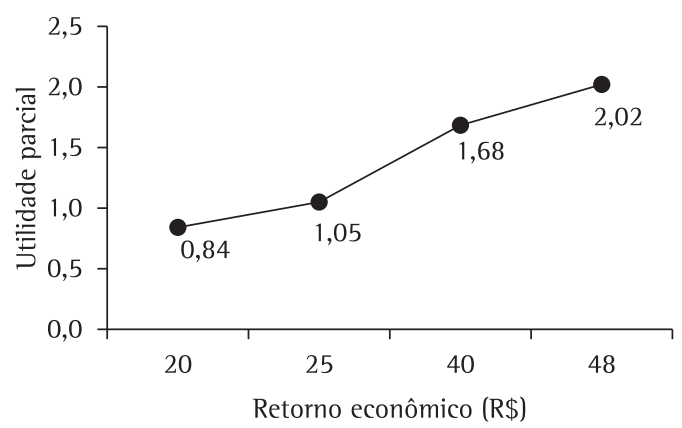

Figura 2. Gráfico das funções de valor de utilidade parciais do retorno econômico. Fonte: elaborado pelos autores.

p. 609): calculam-se os valores das utilidades parciais de cada nível de atributo, com respectivo valor da estimativa do parâmetro $\beta$ calculado para o atributo; calculam-se as importâncias absolutas de cada atributo, por meio da diferença entre o maior valor e o menor valor de utilidade parcial; calculam-se as importâncias relativas, através da normalização das importâncias absolutas.

Para um melhor entendimento desses cálculos, as Tabelas 9 e 10 apresentam os cálculos efetuados para se encontrar os resultados da Tabela 8.

Para interpretar os resultados, é conveniente fazer o gráfico das funções de valor de utilidade parciais, nas quais, no eixo das abscissas $(X)$, tem-se as variáveis independentes e, no eixo das ordenadas $(\mathrm{Y})$, os valores das utilidades que foram calculadas através do modelo matemático. Com a ajuda do gráfico, fica fácil observar e analisar os valores parciais de cada variável e identificar as variáveis mais representativas.

Como pode ser visto na Figura 2, existe uma maior preferência pelo retorno econômico maior ( $R \$ 48,00)$. A segunda preferência foi para o retorno econômico de $\mathrm{R} \$ 40,00$ por saca de $60 \mathrm{~kg}$ e assim sucessivamente. Para os outros atributos, o procedimento é o mesmo. Para se comparar a importância dos vários atributos em termos de importância relativa, deve-se padronizar uma origem, que será a mesma para todos os atributos e, respeitando-se as amplitudes originais, que têm natureza intervalar, assim os novos valores das utilidades são calculados e comparados.

Portanto, os interessados em investir na produção de oleaginosas podem empregar essas variáveis de decisão para a formulação de estratégias e/ou políticas públicas, começando pela análise do primeiro atributo (oleaginosa), seguida da análise do segundo (retorno econômico) e assim até o último, em uma análise vertical. A seguir, deve-se realizar uma análise detalhada dos níveis de cada atributo, para entender o significado dos valores de suas utilidades e os motivos que levaram os respondentes a expressarem tais valores.

\section{Considerações finais}

No processo de tomada de decisão, um dos aspectos mais importantes é a identificação das variáveis que o influenciam. Dessa forma, este estudo buscou identificar a importância e a utilidade dada pelos produtores às diferentes variáveis de decisão condicionantes de investimentos na planta agrícola de oleaginosas no sertão central cearense para a produção de biodiesel, por meio de um modelo de escolha discreta com base em dados de PD. Para tanto, foram utilizadas variáveis que refletem o retorno econômico, o teor de óleo extraído e a existência de assistência técnica rural.

Quanto à importância dada pelos produtores às diferentes variáveis de decisão, a variável retorno econômico apresentou o maior grau de importância, seguida pela variável existência de boa assistência técnica e pelo tipo de oleaginosas. A variável teor de óleo mostrou não ter importância para a decisão dos produtores rurais. Como mencionado antes, isso pode ter sido consequência de o teor de óleo não ser uma variável-chave na tomada de decisão de investimento em oleaginosas ou pode ter havido um problema no delineamento do experimento. 
Quanto às utilidades parciais e à importância de cada atributo e nível de atributo no processo de escolha dos agricultores, ressalta-se que os resultados apresentados refletem a realidade das localidades Canindé, Quixeramobim, Quixadá, Itapipoca e Sobral, não permitindo uma generalização para o perfil do produtor no semiárido brasileiro. No entanto, ficou claro que os atributos que têm maior importância são: retorno econômico e existência de boa assistência técnica rural. Observou-se que não existem correlações fortes entre os parâmetros estimados, o que dá consistência ao modelo encontrado. De acordo com os estudos realizados na revisão de literatura e ao longo das pesquisas de campo, percebe-se que o mercado de biodiesel possui grande potencial de ascensão, visto que sua demanda é garantida por lei específica e os produtores mostraram-se adeptos do cultivo de oleaginosas. Porém, o amadurecimento desse mercado somente será possível se algumas ações forem realizadas.

Em primeiro lugar, é necessário a implantação de programas de assistência técnica e treinamento (existência de assessoria de nível superior, parcerias com Centec e assistência da Embrapa) que serão fundamentais para capacitar e orientar os agricultores no correto manejo das oleaginosas. Somado a isso, as Ater podem também combater os preconceitos existentes com relação ao cultivo da mamona, do algodão e de outras culturas no sertão central cearense.

Uma segunda ação seria a avaliação da capacidade de produção de oleaginosas no país, de acordo com a vocação regional, prevendo-se a expansão de áreas das oleaginosas com domínio tecnológico, incentivando o extrativismo sustentável de espécies nativas existentes em reservas naturais e o cultivo de oleaginosas perenes.

Deve-se ressaltar a importância do incentivo à agricultura familiar, já que esta é peça fundamental para a expansão da área das oleaginosas, pois é a que agrega renda a grande parte da população rural. 0 agricultor familiar tem a mensuração de sua atividade não mais pela área física que possui ou ocupa, mas pela renda que consegue extrair de cada hectare dessa área ou de cada animal que produz. Essa nova realidade coloca em xeque os conceitos tradicionais de agricultura familiar e empresarial, exigindo uma nova postura dos agentes econômicos envolvidos na atividade primária.

Este estudo apresentou algumas limitações. Contudo, a existência de tais limitações não invalida os resultados encontrados. As limitações encontradas dão origem a sugestões para estudos futuros. À medida que for possível coletar um volume maior de dados primários, haverá a possibilidade de ampliar o escopo do trabalho. Uma das sugestões é aplicar o mesmo estudo para outros municípios e estados brasileiros. Assim será possivel identificar a influência das diversas variáveis em cada estrato e em cada região do Brasil, captando suas especificidades.

\section{Referências}

ALVES, B. B. A importância da variabilidade do tempo de viagem no acesso terrestre a aeroportos: um estudo de caso do aeroporto internacional André André Franco Montoro. 2005. 160 f. Dissertação (Mestrado em Engenharia de Transportes)-Escola Politécnica, Universidade de São Paulo, São Paulo, 2005.

ANTUNES, J.; SANTOS, E. S. Análise da Decisão. In: CORRAR, L. J.; TEÓPHILO, C. R. Pesquisa Operacional para decisão em contabilidade e administração: contabilometria. São Paulo: Atlas, 2009.

ARRUDA, J. B. F. Evaluation of Urban Transport Projects in Developing Countries: An Accessibility Approach. 1995. Thesis (Doctorate)-Institute for Transport Studies, School of Business and Economic Studies, The University of Leeds, United Kingdom,1995.

BEN-AKIVA, M.; LERMAN, S. Discrete Choice Analysis: theory and application to travel demand. 7th ed. Boston: MIT Press, 1985.

BENTHAN, J. An introduction to the principles of morals and legislation. Kitchener: Batoche Books, 1781. Disponível em: <www.efm.bris.ac.uk/het/bentham/morals.pdf>. Acesso em: 16 out. 2006.

BRANDÃO FILHO, J. E. Previsão de demanda por gás natural veicular: uma modelagem baseada em dados de preferência declarada e revelada. 2005. 274 f. Dissertação (Mestrado em Engenharia de Transportes)-Universidade Federal do Ceará, Fortaleza, 2005.

BRASIL. Ministério do Desenvolvimento Agrário. BIODIESEL. Disponivel em: <http://www.biodiesel.gov.br>. Acesso em: 02 nov. 2007.

BRASIL. Presidência da República. Lei no 11.097. Dispõe sobre a introdução do biodiesel na matriz energética brasileira; altera as Leis $n^{\circ}$ 9.478, de 6 de agosto de 1997, $n^{\circ}$ 9.847, de 26 de outubro de 1999 e $n^{\circ} 10.636$, de 30 de dezembro de 2002; e dá outras providências. Diário Oficial da República Federativa do Brasil, Brasília, DF, 13 jan. 2005. Disponível em <http://www.planalto.gov.br/ ccivil/_Ato2004-2006/2005/Lei/L11097.htm>. Acesso em: 05 mar. 2008.

BRIGHAM, E. F.; EHRHARDT, M. C. Financial management: theory and practice. South-Western: Thomson Learning, 2002.

BRITO, A. N. Aplicação de um procedimento usando Preferência Declarada para a estimativa do valor do tempo de viagem de motoristas em uma escolha entre rotas rodoviárias pedagiadas e não pedagiadas. 2007. $185 \mathrm{f}$. Dissertação (Mestrado em Engenharia de Transportes)Escola Politécnica, Universidade de São Paulo, São Paulo, 2007.

CARMO, H. C. E.; DIAZ, M. D. M. Análise da base de dados e utilização de variáveis binárias (dummy). In: VASCOLCELOS, M. A. S.; ALVES D. Manual de econometria: nível intermediário. São Paulo: Atlas, 2000.

CERVO, A. L.; BERVIAN, P. A. Metodologia científica: para uso dos estudantes universitários. São Paulo: McGrawHill do Brasil, 1983. 
CHEN, K.; XIAO, T. Demand disruption and coordination of the supply chain with adominant retailer. European Journal of Operational Research, v. 197, p. 225234, 2009. http://dx.doi.org/10.1016/j.ejor.2008.06.006

FREITAS, A. A. F. Modelagem comportamental dos decisores através de técnicas de preferência declarada: uma aplicação no setor imobiliário de FlorianópolisSC. 1995. Dissertação (Mestrado em Engenharia de Produção)-Universidade Federal de Santa Catarina, Florianópolis, 1995.

GUIMARÃES, L. G. A. Tomada de decisão em investimentos na produção de oleaginosas para o setor de biodiesel, com foco na pequena e média empresa: uma abordagem de análises discriminante e fatorial. 2008. 122 f. Dissertação (Mestradoem Logística e Pesquisa Operacional)Universidade Federal do Ceará, Fortaleza, 2008.

HAAIJER, R.; KAMAKURA, W.; WEDE, M. Response latencies in the analysis of conjoint choice experiments. Journal of Marketing Research, v. 37, n. 3, p. 376-382, 2000. http:// dx.doi.org/10.1509/jmkr.37.3.376.18775

HAGUE CONSULTING GROUP - HCG. Software ALOGIT 4.1 Licensed by FCPC Brasil. The Netherlands: HCG, 2004.

HAIR JUNIOR, J. F. et al. Análise Multivariada de dados. 5. ed. Porto Alegre: Bookman, 2005.

HENSHER, D. A. Stated preference analysis of travel choices: The state of practice. Transportation, V. 21, n. 2, p. 107-133, 1994.

KROES, E. P.; SHELDON, R. J. Stated preference methods: an introduction. Journal of Transport Economics and Policy, v. 22, n. 1, p. 11-25, 1988.

LOUVIERE, J. J.; HENSHER, D. A.; SWAIT, J. D. Stated Choice Methods: Analysis and Application. Cambridge: Cambridge University Press, 2000. http://dx.doi. org/10.1017/CB09780511753831

MALHOTRA, N. K. Pesquisa de Marketing: Uma Orientação Aplicada. 4. ed. São Paulo: Bookman, 2006.

MENDES, R. A. Diagnóstico, Análise de Governança e Proposição de Gestão para a Cadeia Produtiva do Biodiesel da Mamona (CP/BDM): o Caso do Ceará. 2005. 159 f.
Dissertação (Mestrado em Engenharia de Transportes)Universidade Federal do Ceará, Fortaleza, 2005.

MORIKAWA, T. Incorporating stated preference data in travel demand analysis. 1989. Thesis (Doctorate)-Massachusetts Institute of Technology, Boston, 1989.

MONTGOMERY, D. C. Design and analysis of experiments. 5th ed. New York: John Wiley \& Sons, 2001.

ORTÚZAR, J. D.; WILLUMSEN, L. G. Modelling Transport. 3rd ed. New York: John Wiley \& Sons, 1994.

PEDRET, R.; SAGNIER, L.; CAMP, F. Herramientas para segmentar mercados y posicionar productos: análisis de información cuantitativa en investigación comercial. Barcelona: ED, 2000.

PERES, J. R. R.; BELTRÃO, N. E. M. Oleaginosas para biodiesel: situação atual e potencial. In: FERREIRA, J. R.; CRISTO, C. M. P. N. O Futuro da Indústria: Biodiesel. Brasília: Governo, 2006.

SENNA, L. A.S. User's Response to Travel Time Variability. 1994. Thesis (Doctorate)-Institute for Transport Studies, The University of Leeds, United Kingdom, 1994.

\section{Agradecimentos}

Agradecemos as sugestões dos revisores, que contribuíram para a qualidade da metodologia, nos ajudando a perceber a importância do detalhamento da modelagem multinomial Logit, mais especificamente da função de verossimilhança utilizada; da análise dos dados e interpretação dos resultados; das conclusões; e da redação geral do trabalho. Agradecemos também ao Departamento Nacional de Obras Contra as Secas (DNOCS) e ao grupo de trabalho Biodiesel com Inclusão Social no estado do Ceará, por nos terem permitido participar de todas as atividades do grupo, bem como por terem estruturado o nosso encontro com os agricultores familiares das principais regiões produtoras de oleaginosas do Ceará.

\title{
Stated preference analysis to study the utility of oilseeds production
}

\begin{abstract}
The aim of the present research was to perform a discreet choice preference analysis model and assess the oilseeds offer, through Stated Preference data, considering that the Brazilian Law 11.097/2005 establishes compulsory introduction of biodiesel in the Brazilian energy matrix, guaranteeing its demand. Descriptive survey methodology was used. The universe of this study comprised median and small national producers, adding up to a sample of 114 valid answered questionnaires. Quantitative model with Stated Preference Analysis of data treatment was used. Among the main results of this study, it was possible to verify that the most important attributes were economic return and existence of satisfactory national technical assistance. The variable extracted oil content is presented as unimportant to national producers' choice. A suggestion for further studies is the application of similar researches to other Brazilian cities and States.
\end{abstract}

\section{Keywords}

Logistic. Oilseeds. Biodiesel. Stated preference. 